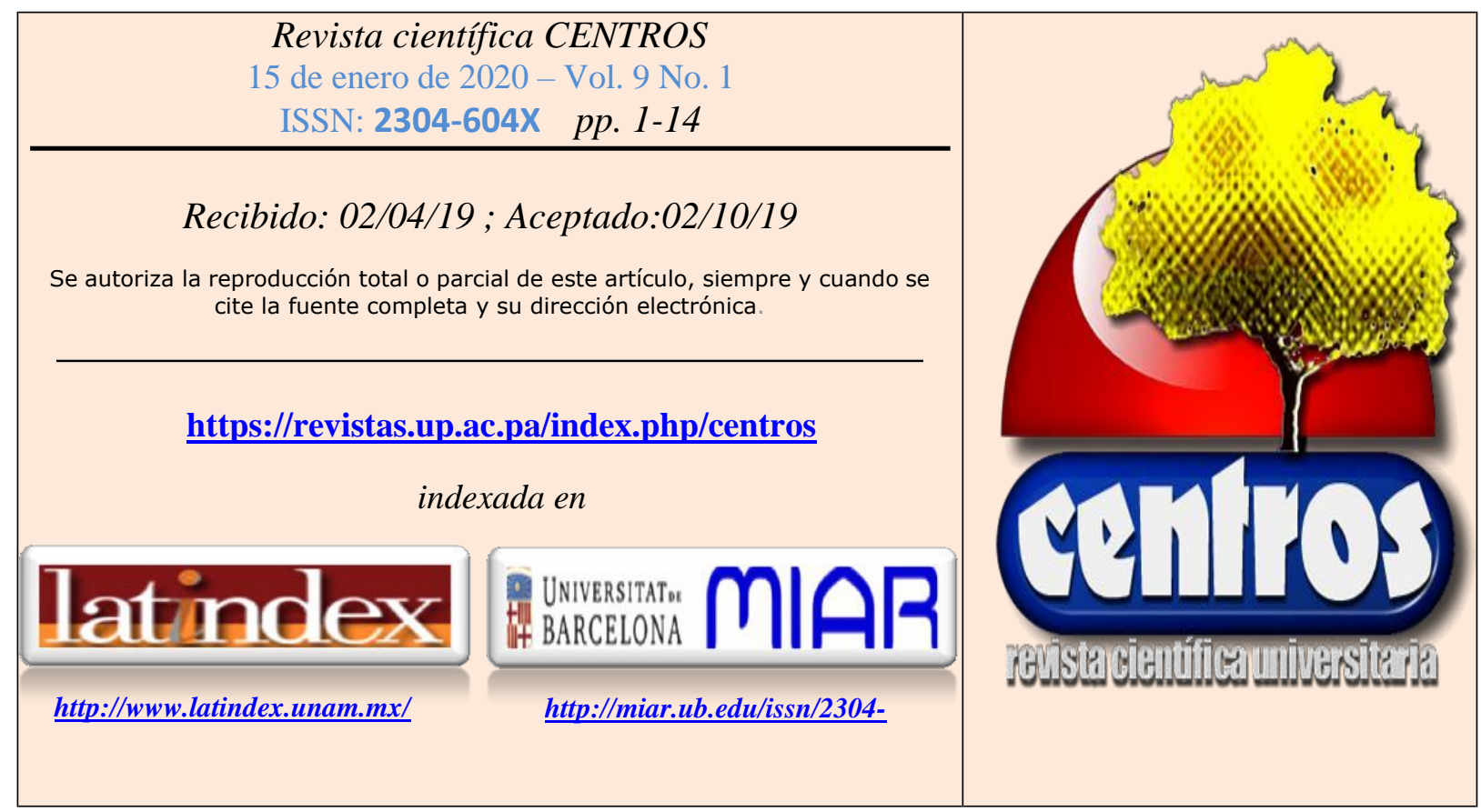

\title{
FOMENTO DE LA TRIBUTACIÓN DESDE LA VINCULACIÓN CON LA SOCIEDAD. CASO UNEMI
}

\section{PROMOTION OF TAXATION FROM THE LINK WITH SOCIETY. UNEMI CASE}

\author{
Ana Carvajal, Yecenia Escobar, María Fernanda Moreira \\ Universidad Estatal de Milagro \\ acarvajals1@unemi.edu.ec, yescobard@unemi.edu.ec, mmoreiram4@unemi.edu.ec
}

\section{RESUMEN}

Este trabajo tiene por objetivo exponer las estrategias aplicadas en el proyecto de vinculación Consultoría tributaria y contable en la comunidad 2018 de la UNEMI, que permitieron conocer la realidad situacional de los contribuyentes. A través del proyecto de vinculación se busca formar a comerciantes, personas naturales no obligadas a llevar contabilidad bajo relación de dependencia, y miembros de las asociaciones y cooperativas agrícolas de la ciudad de Milagro y sus zonas aledañas, con el propósito de crear conciencia de sus obligaciones con el Estado, fomentando una cultura tributaria y manejo de registros contables básicos. Las estrategias aplicadas para la ejecución del proyecto son: planificación, preparación de los estudiantes, capacitación a los contribuyentes y análisis de los resultados. La investigación es exploratorio-descriptiva y de campo, los beneficiarios directos están representados por 323 personas. Para la recolección de la información se aplicó la técnica de la encuesta. Los resultados preliminares revelaron que las asociaciones recurren a terceros para cumplir con sus deberes formales, pero después de recibir las capacitaciones se evidencia un mayor dominio en temas tributarios. Para lograr una formación tributaria que conduzca al ciudadano a cumplir sus responsabilidades con el estado, se considera necesario el trabajo en conjunto entre la administración tributaria, instituciones de educación superior y la comunidad. 
Palabras claves: vinculación con la sociedad, tributación, administración tributaria, UNEMI

\begin{abstract}
This paper aims to expose the strategies applied in the tax consulting and accounting consulting project in the 2018 community of UNEMI, which allowed to know the situational reality of taxpayers. Through the linking project seeks to train traders, natural persons not obligated to maintain accounting records under dependency relationship, and members of agricultural associations and cooperatives in the city of Milagro and its surrounding areas, with the purpose of raising awareness of their obligations to the State, promoting a tax culture and management of basic accounting records. The strategies applied to the execution of the project are: planning, training of the students, training of the taxpayers and analysis of the results. This paper is exploratory-descriptive and field, direct beneficiaries are represented by 323 people. To collect the information, the survey technique was applied. The preliminary results revealed that the associations resort to third parties to comply with their formal duties, but after receiving the training, a greater mastery in tax issues is evident. Finally, it was possible to conclude that to achieve a tax training that leads the citizen to fulfill their responsibilities with the state, it is considered necessary to work together between the tax administration, higher education institutions and the community.
\end{abstract}

Keywords: Linkage with society, taxation, tax administration, UNEMI

\title{
INTRODUCCIÓN
}

Los impuestos son tributos de mucha importancia para la economía de un país, por lo ingresos fiscales que generan para cubrir las necesidades sociales y promover el desarrollo económico, por lo tanto, es importante inducir al contribuyente al cumplimiento voluntario de sus obligaciones tributarias.

En Ecuador a inicios del siglo XXI con la dolarización de la economía, los tributos pasaron a ser la principal fuente de ingresos para el presupuesto general del estado, de ahí que surgen nuevas medidas impositivas. En la actualidad el número de contribuyentes ha aumentado de 82.458 en el 2015 a 196.459 en el 2018 (Servicio de Rentas Internas, 2018), por la obligatoriedad que tienen de inscribirse en el Registro Único del Contribuyente (RUC) todas las personas naturales que inicien o realicen actividades económicas, sin embargo, aún persisten los negocios informales y con ello, resistencia al momento de cumplir sus obligaciones fiscales; situación que perjudica al Servicios de Rentas Internas (SRI), al afectar los ingresos previstos para el período fiscal.

Es así que, el Estado, a través del Plan Nacional de Desarrollo, 2017 - 2021 "Toda una Vida", marca un cambio en el rumbo de la educación superior, llevándola a asumir un compromiso con la sociedad, esto implica que los futuros profesionales deben brindar sus conocimientos en beneficio de la colectividad, demostrando que saben hacer y compartir, generando un verdadero saber vivir. El Plan Nacional de desarrollo dentro del eje 2 toma como premisa que la economía está al servicio de la sociedad, por ello, en su objetivo 4 buscar consolidar la sostenibilidad del sistema económico, social y solidario, y afianzar la dolarización, proponiendo medidas de prevención de la elusión y evasión fiscal que permitan incrementar la recaudación, fortalecer la eficiencia y profundizar la progresividad del sistema tributario (Secretaría Nacional de Planificación y Desarrollo, 2017). Para lograr este propósito se busca acuerdos de cooperación entre la 
administración tributaria y las instituciones educativas que fomenten capacitaciones en temas tributarios (Onofre-Zapata, Aguirre-Rodriguez, \& Murillo-Torres, 2017).

Por su parte, la Cepal, (2018) a través de la agenda 2030 para el Desarrollo Sostenible, pondrá énfasis en las siguientes prioridades:

- El análisis de aspectos clave del financiamiento para el desarrollo, incluido el combate a los flujos ilícitos y la reducción de la evasión y elusión fiscales

- El fortalecimiento de la acción e integración regionales en las áreas productiva, comercial, tecnológica, fiscal, financiera, de infraestructura y en las cadenas de valor.

- La reafirmación de la relevancia de las instituciones y la redefinición de la ecuación entre el Estado, el sector privado y la sociedad civil.

Así también la Cepal, (2018) en sus Objetivos de Desarrollo Sostenible (ODS) busca reducir la desigualdad en los países y entre ellos. Por tal razón, una de las Metas del Objetivo 10, es adoptar políticas, especialmente fiscales, salariales y de protección social, y lograr progresivamente una mayor igualdad.

Por tal razón, la Universidad Estatal de Milagro (UNEMI), como centro de educación superior público llevó a cabo el proyecto de vinculación denominado "Consultoría tributaria y contable a la comunidad, 2018" permitiendo la interacción con la sociedad como un proceso educativo en donde las carreras se acerquen principalmente a zonas rurales o urbano marginales.

La investigación es exploratoria, descriptiva, evaluativa y de campo. Se utilizó la técnica de la encuesta para la recolección de la información. Las estrategias aplicadas para el desarrollo del proyecto son: 1) planificación, 2) preparación de los estudiantes, 3) capacitación a los contribuyentes y 4) análisis de los resultados. Estas fases permitieron observar el bajo nivel de cultura tributaria y de gestión administrativa-contable que mantienen los beneficiarios, lo que genera que los contribuyentes no cumplan sus obligaciones correctamente afectando a los ingresos previstos del Estado para el período fiscal. El estudio se aplicó a 323 miembros agremiados; el periodo de análisis es 2018, año en el cual se efectúo la vinculación con la colectividad.

El trabajo se divide en cuatro partes: primero una revisión literaria, en el cual constarán las teorías y conceptualizaciones más relevantes sobre el tema, en la segunda sección, se describe la metodología a aplicar para el desarrollo del trabajo, en tercer lugar, se encuentran los procedimientos que se aplicaron para la obtención de la información, en cuarto lugar, el análisis de los resultados y, finalmente, la conclusión y recomendación del trabajo.

\section{MARCO TEÓRICO}

\section{Vinculación con la sociedad desde las normativas nacionales e internacionales}

Los pilares fundamentales que tiene la universidad para llevar a cabo los programas de vinculación se asientan sobre los principios de la Conferencia Mundial de la Educación Superior (Paris, 1998), que en su artículo 6 declaró que entre las funciones y misiones de la educación superior se debe evaluar la pertinencia en función de lo que la sociedad espera de la institución y lo que estas hacen, siendo necesario reforzar sus funciones de servicio a la sociedad. Así mismo, en su artículo 2 menciona que las entidades de educación superior deben contribuir con la solución de los problemas que afectan a las comunidades, 
las naciones y la sociedad mundial, y en el artículo 17 hace hincapié en que "la asociación basada en el interés común, el respeto mutuo y la credibilidad deberá ser una modalidad esencial para renovar la enseñanza superior" (UNESCO, 1998)

La interacción entre las universidades y la sociedad también se encuentra enmarcada en el proyecto denominado Metas Educativas 2021: la educación que queremos para la generación de los Bicentenarios, que nació en el Salvador por iniciativa de los ministros de Educación Iberoamericanos; donde menciona que es necesario "elevar la participación de los diferentes sectores sociales y su coordinación en proyectos educativos: familias, universidades y organizaciones públicas y privadas", sobre todo de aquellas relacionadas con la promoción del desarrollo económico, social y cultural y su nivel de logro está valorado por el número de proyectos, en los que participan varios sectores sociales (CEPAL, OEI, \& Iberoamericana, 2010).

En Ecuador, la vinculación con la sociedad se asienta en cuatro normas:

1) La constitución que promueve la educación superior pública y gratuita, la investigación y "la construcción de soluciones para los problemas del país, en relación con los objetivos del régimen de desarrollo" (Art. 350 Constitución de la Republica de Ecuador).

2) El Plan Nacional de Desarrollo 2017-2021 que establece políticas, objetivos y estrategias, con la finalidad de crear una sociedad orientada hacia un nuevo régimen de desarrollo que sea inclusivo, equitativo y solidario.

3) La Ley Orgánica de Educación Superior (LOES), replantea la educación como bien público y motor de desarrollo, toda vez que el papel de las universidades se valora de acuerdo a sus aportes en el desarrollo social. En el Art. 8 menciona que dentro de los fines de la educación superior se encuentra "Contribuir en el desarrollo local y nacional de manera permanente, a través del trabajo comunitario o extensión universitaria". Por su parte, en el Art. 87 señala: "Como requisito previo a la obtención del título, los y las estudiantes deberán acreditar servicios a la comunidad mediante prácticas o pasantías preprofesionales, debidamente monitoreadas, en los campos de su especialidad"

4) Los modelos para la evaluación de la calidad de las instituciones educativas y los programas académicos diseñados por el Consejo de Evaluación, Acreditación y Aseguramiento de la Calidad de la Educación Superior.

En la UNEMI, los proyectos de vinculación forman parte del Plan Estratégico de Desarrollo Institucional (PEDI) 2018-2021, que establece que todos los programas de vinculación se enfocaran en el desarrollo regional desde las comunidades hasta la región 5, articulados con:

- Los ODS y el desarrollo regional;

- Los objetivos del PND y el desarrollo regional;

- Los GAD's, el sector público y el desarrollo regional;

- El sector privado, la economía popular y solidaria y el desarrollo regional; y,

- La educación continua y el desarrollo regional;

Cumpliendo con este conjunto de normas, a las entidades de educación superior les corresponde la interacción con la sociedad como un proceso educativo en donde las carreras se acerquen a la comunidad, principalmente en zonas rurales o urbano marginales. Esto garantiza un proceso formativo en los estudiantes donde a través de la transferencia de saberes compartidos, se beneficia directa e indirectamente a la comunidad creando procesos de transformación social 


\section{Cultura tributaria}

La cultura tributaria se entiende como el conocimiento y cumplimiento voluntario por parte del contribuyente de las obligaciones tributarias que permiten la realización de obras en beneficio de la población, siendo necesario profundizar la relación que debe existir entre el Estado y el ciudadano.

Uno de los objetivos de la educación fiscal es crear consciencia sobre la responsabilidad de cumplir con los deberes formales, como un deber y no como una obligación. Para fortalecer la cultura tributaria es necesario que los contribuyentes tengan conocimientos sólidos sobre las disposiciones tributarias. Estos conocimientos se obtienen con la transferencia de información de forma oportuna, práctica y sencilla, permitiendo de esta forma, una recaudación sostenible a largo plazo. El SRI tiene por finalidad consolidar la cultura tributaria que permita incrementar la recaudación a través del cumplimiento consciente de sus responsabilidades tributarias por parte del contribuyente.

Quintanilla, (2012) menciona que:

Teniendo presente que la Constitución se refiere a la persona como titular de derechos y obligaciones, se define a la cultura tributaria como el conjunto de costumbres y hábitos individuales y colectivos que permiten el cumplimiento de los deberes y la defensa de los derechos relacionados con los tributos pagados al estado. En este sentido no sólo el ciudadano está llamado a tener conciencia de sus obligaciones como contribuyente, sino también el estado, cumpliendo con las obras y servicios financiados con el dinero recaudado (p. 110)

Por su parte, (Camarero, Pino, \& Mañas, 2015) argumentan que "por cultura tributaria se entiende al universo simbólico que sustenta el pago de impuestos, es decir, el conjunto de creencias, sentimientos y comportamientos que se implican en la institución social de la recaudación de impuestos" (p. 99). Amasifuen, (2015) menciona que la cultura tributaria no solo implica conocer las obligaciones con el fisco, sino también cumplir con los deberes tributarios, es decir, la población debe entender lo que debe hacer y lo que no, en el pago de impuesto. Es claro que los países desarrollados tienen mayores niveles de cultura tributaria por su grado de satisfacción al ver sus recursos cristalizados en obras y servicios públicos.

Para lograr una formación tributaria sólida que conduzca al ciudadano hacia la formalidad y cumplimiento de sus responsabilidades, se considera necesario el trabajo en conjunto entre la administración tributaria, instituciones de educación superior y la comunidad. Si existe cooperación se logrará la cultura tributaria que necesita el país.

La universidad ecuatoriana es de carácter humanista, cultural y científica (Quintanilla, 2012), en ella se reconoce la importancia de fortalecer y defender al ser humano integrante de cada grupo social y comunidad en general. Tiene como rol buscar el bien común de la sociedad. La universidad ecuatoriana y sus planes conjuntos de educación tributaria surgen como parte de su responsabilidad social, es decir, "estar consciente de que la relevancia de su trabajo será evaluada en función de su auténtico compromiso social (...) priorizando, por razones éticas, el empoderamiento de los sectores más desfavorecidos" (Van Der Bijl, 2015 p. 14). Entendiendo que su función no es solo impartir conocimientos en temas tributarios sino también contribuir a fortalecer la cultura tributaria.

Todo ciudadano sea este comerciante, profesional, en relación de dependencia, agremiado, entre otros, tiene la responsabilidad social y moral de cumplir con sus 
obligaciones tributarias, por eso, es importante la realización de capacitaciones enfocadas de forma práctica y sencilla para cada sector económico.

En el Código Tributario emitido en el Registro Oficial Suplemento 38 y modificada el 21 de agosto de 2018, se enumeran los siguientes deberes formales del contribuyente:

- Inscribirse en los registros pertinentes, proporcionando los datos necesarios relativos a su actividad; y, comunicar oportunamente los cambios que se presenten.

- Emitir y entregar comprobantes de venta autorizados.

- Llevar los libros y registros contables relacionados con la actividad económica.

- Presentar las declaraciones que correspondan y pagar los impuestos.

- Concurrir a las oficinas de la administración tributaria, cuando su presencia sea requerida por autoridad competente.

El estado tiene como obligación crear confianza en el pueblo y mejorar la distribución equitativa de los recursos a través de la política pública, por ello, tiene la facultad de crear y derogar las disposiciones legales que permitan el cumplimiento del fin mayor, que es, la recaudación en cada período de los ingresos para financiar el gasto público.

En la actualidad, el país cuenta con microempresarios que aportan a la economía, pero enfrentan el problema de falta de información dando como resultado baja cultura tributaria. Para evitar el incumplimiento de los deberes formales y reducir la informalidad, el SRI creo el Régimen Impositivo Simplificado Ecuatoriano (RISE), que busca promover la cultura tributaria en el país (Arriaga Baidal, Reyes Tomalá, Olives Maldonado, \& Solórzano Méndez, 2017). El RISE permite a los negocios informales incorporarse al cumplimiento de las disposiciones legales y reemplaza el pago de impuesto a la renta, del Impuesto al valor agregado (IVA) y las declaraciones, por el pago de cuotas mensuales.

En Ecuador la informalidad es considera como fuente de poca recaudación y de acuerdo al Instituto Ecuatoriano de Estadísticas y Censos (INEC), el sector informal se define como "el conjunto de personas ocupadas que trabajan en establecimientos que se no encuentran registrados en el RUC". En junio del 2018, el 47.5\% a nivel nacional corresponde al sector informal, mientras que para septiembre del 2018 este porcentaje es del 46.2\% (INEC, 2018). En Milagro de acuerdo a la Figura 1, la informalidad ha disminuido progresivamente por las capacitaciones y campañas de la Administración tributaria para impulsar en los contribuyentes su inscripción en el RUC.

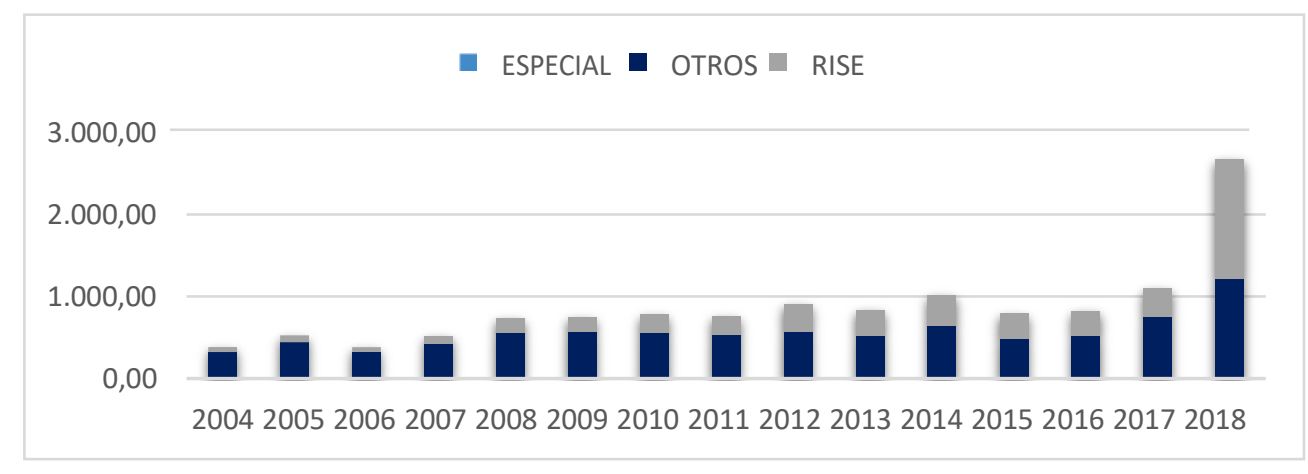

Figura 1. Contribuyentes inscritos en el RUC y RISE de Milagro Fuente: https://declaraciones.sri.gob.ec/saiku-ui/

Ante estos antecedentes surge la imperiosa necesidad de fomentar los conocimientos tributarios y contables en la comunidad, para un mejor cumplimiento de las obligaciones que demandan los Organismos de Control y que contribuirán a incrementar la recaudación fiscal 
en Milagro y sus zonas de influencia.

Es compromiso de las instituciones del sector público incluir dentro de su planificación programas de vinculación con la sociedad. Es así que, las universidades impulsan capacitaciones masivas a la sociedad en general convirtiéndose en pieza clave para el mejoramiento y fortalecimiento de los conocimientos tributarios, aportando en la formación y motivando a los comerciantes, profesionales y estudiantes a través de información oportuna y adecuada, e impulsándolos a asumir la responsabilidad que conllevan el pago de sus obligaciones fiscales en beneficio del país y de sus negocios, contribuyendo con la disminución de la falta de interés, desconocimiento de las leyes y con el cumplimiento voluntario y consciente de las obligaciones tributarias.

\section{MATERIALES Y METODOS}

El proyecto de vinculación con la colectividad en temas tributarios - contables de la UNEMI nace ante la necesidad de que las instituciones de educación superior cumplan con su función, como generadores de conocimiento para resolver problemas de la sociedad, llevando alternativas de desarrollo y dando también a sus educandos la oportunidad de realizar sus prácticas con sus conocimientos. A través del proyecto "Consultoría tributaria y contable a la comunidad, 2018" se promueve el aprendizaje colectivo entre los estudiantes, docentes y la comunidad. Para ello, involucra a los estudiantes de sexto y séptimo semestre de la carrera de Contaduría Pública y Auditoría. El trabajo se desarrolla en dos fases, la I fase involucra el análisis de la situación actual de los contribuyentes, la planificación de las actividades a realizar y formación de los contribuyentes. En la II fase, se pretende medir los resultados de la formación tributaria. Se identificó 323 beneficiarios en el período 2018.

La metodología del proyecto es de carácter exploratoria, descriptiva y evaluativa, y aplica una investigación de campo, porque se hicieron observaciones in-situ a través de encuestas para examinar a los contribuyentes. El procesamiento de los datos se estructuró en formato tabular para analizar la información de los beneficiarios del proyecto. Las estrategias aplicadas para el acercamiento con los miembros de las asociaciones, comerciantes y personas naturales bajo relación de dependencia, fueron: Planificación, capacitaciones a los estudiantes, capacitaciones a los contribuyentes y análisis de los resultados.

\section{Planificación}

Antes de la ejecución del proyecto se analizó la problemática de la comunidad y se determinó que existía desconocimiento en materia tributaria por parte de los beneficiarios del proyecto (Figura 2), mediante visitas y llamadas telefónicas. Los miembros de las asociaciones cumplían con el requisito de realizar una actividad económica, ser personas naturales no obligadas a llevar contabilidad y evidenciar a través de las encuestas la falta de conocimientos en temas tributarios.

Luego de analizar la problemática se creó el proyecto con el objetivo de formar a comerciantes, personas naturales no obligadas a llevar contabilidad bajo relación de dependencia, y miembros de las asociaciones y cooperativas agrícolas de la ciudad de Milagro y sus zonas aledañas, con el propósito de crear conciencia de sus obligaciones con el Estado, fomentando una cultura tributaria y manejo de registros contables básicos. 


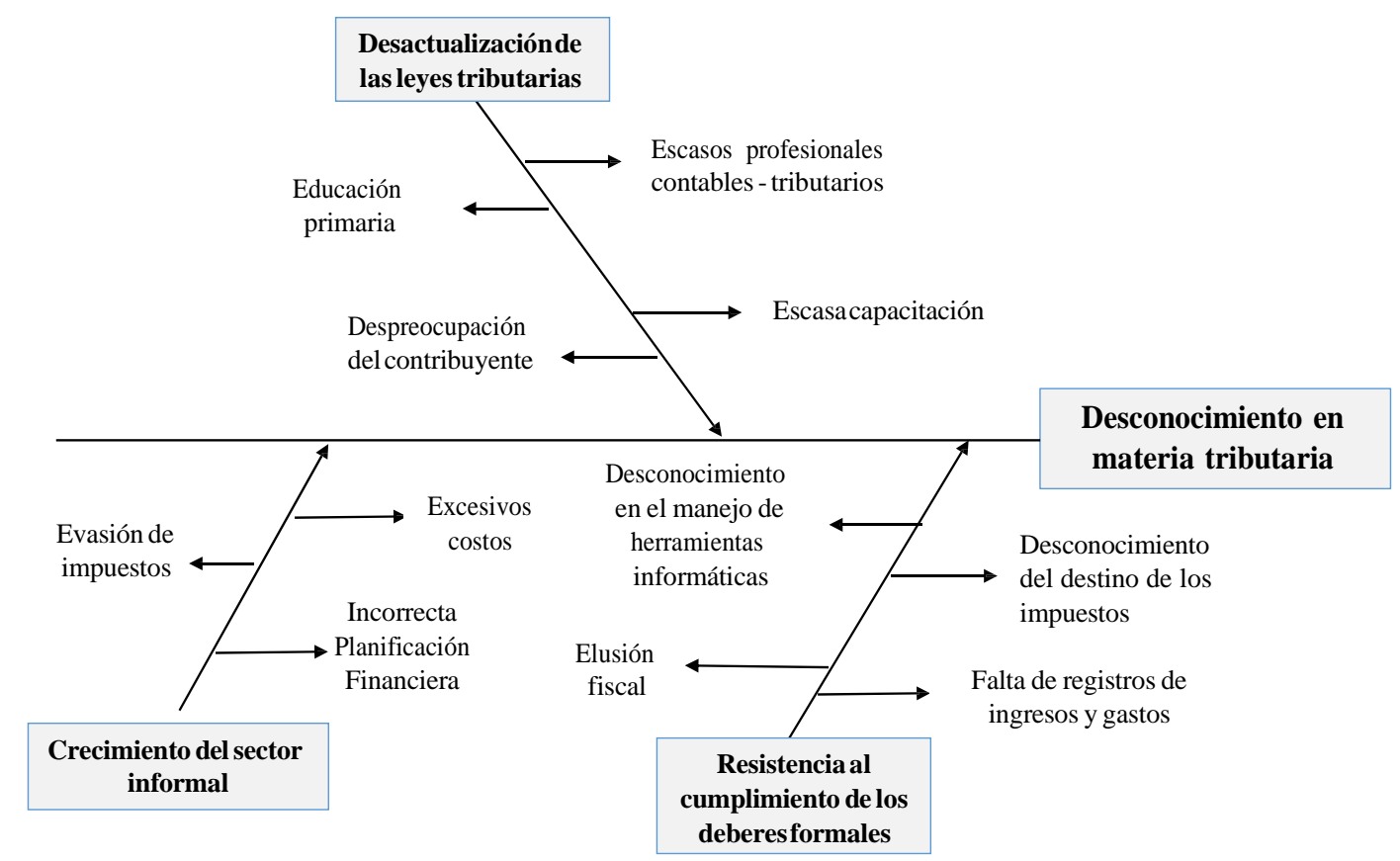

Figura 2. Espina de pescado - causas

Fuente: Proyecto de vinculación

En la Tabla 1, se muestra a los beneficiarios del proyecto, quienes siempre mostraron su colaboración y dedicaron tiempo para formarse en temas básicos de tributación. Las asociaciones pertenecen a los cantones de: Milagro, Yaguachi, San Carlos, Roberto Astudillo, Mariscal Sucre y Naranjito, que suman un total de 15 agrupaciones beneficiarias. Cabe indicar que la capacitación fue impartida a los integrantes de las asociaciones o gremios, que de forma individual tienen obligaciones con el fisco.

Tabla 1. Identificación de los beneficiarios del proyecto

\begin{tabular}{ll}
\hline \multicolumn{1}{c}{ Asociaciones Identificadas } & \multicolumn{1}{c}{ Cantón } \\
\hline Anda Aguirre & Milagro \\
Asociación Agropecuaria General Pedro J. Montero & Yaguachi \\
Asociación Agropecuaria Maravillas Dos & Milagro \\
Asociación de Agricultores Mariscal Sucre (Asgrimasu) & Mariscal Sucre \\
Asociación de Emprendedores de producción y comercialización "Nueva Era” & Roberto Astudillo \\
Asociación de productores agropecuarios La Lejía "ASPROLEJ”" & Mariscal Sucre \\
Asociación de Trabajadores autónomos "La Paquita" & Naranjito \\
Cooperativa de producción agrícola La Indiana & San Carlos \\
Junta Cantonal de Defensa del Artesano & Milagro \\
Mercado Central de Milagro & Milagro \\
Otto Arosemena Gómez & Milagro \\
Sindicato General de Trabajadores de la Compañía Tropical Packing S.A & Milagro \\
Unidad Educativa "Liceo Cristiano Milagro" & Milagro \\
Unidad Educativa "Río Amazonas" & Naranjito \\
Unidad Educativa Guillermo Durán Arcentales & Roberto Astudillo \\
\hline
\end{tabular}

Fuente: Informes mensuales de avances del proyecto de vinculación

\section{Preparación de los estudiantes}

La Universidad Estatal de Milagro y el SRI, acuerdan mediante la firma de un "Convenio de Colaboración Mutua" apoyar a estos sectores con capacitaciones, además de guiarlos en problemas tributarios. Fruto de este convenio se solicita a funcionarios del SRI 
Agencia Milagro, capacitaciones para los docentes y estudiantes de los últimos semestres de la carrera de Contaduría Pública y Auditoría. La capacitación permitió la elaboración del material y los talleres a ser utilizados durante la vinculación. La capacitación tributaria tuvo una duración de 20 horas, permitiendo despejar dudas y afianzar los conocimientos en los estudiantes.

\section{Capacitación a los contribuyentes}

Se formaron grupos de trabajo de máximo 7 estudiantes, a quiénes se les asignó una asociación. Los estudiantes capacitadores trataron sobre las siguientes temáticas: Deberes Formales, RUC-RISE, Comprobantes de ventas, Impuesto al valor agregado, gastos personales, declaración de impuesto a la renta, Impuesto ambiental, devolución de IVA tercera edad y discapacitados y registros contables básicos. Un total de 85 estudiantes fueron asignados a la capacitación de los miembros de estos sectores vulnerables. El desarrollo de las capacitaciones se dio bajo el acompañamiento de docentes con experiencia en estos temas.

\section{RESULTADOS}

Al finalizar el proceso de capacitación, por un lado, se cumplió con los resultados de aprendizaje de la carrera y se desarrolló en los estudiantes el perfil profesional propuesto y demandado por el mercado laboral y la sociedad, sin olvidar el humanismo, la responsabilidad social y la ética, aspectos que diferencian y marcan una ventaja competitiva en los nuevos profesionales.

Por otro lado, se aplicó una encuesta y pruebas de conocimiento antes y después de la capacitación, a los miembros de las asociaciones calificados como personas naturales no obligadas a llevar contabilidad, para determinar el efecto de la gestión de los estudiantes. De los cual se evidencio que un total de 323 beneficiarios directos se han formado en materia tributaria, generando 1292 beneficiarios indirectos. Es importante señalar que los beneficiarios directos están compuestos por el conjunto de personas que conforman el grupo objetivo al que están dirigidas las acciones del proyecto, mientras que los beneficiarios indirectos son las personas favorablemente impactadas por el beneficio que recibe el grupo objetivo, ya sea porque disfrutan de mayores recursos o servicios, o porque la mejora en la condición de los beneficiarios directos también repercute en ellos.

El número de beneficiarios indirectos es calculado mediante los beneficiarios directos y estos a su vez afectados por el número de personas por vivienda (4 personas) según la última encuesta al 2010 del Censo de Población y Vivienda del INEC. En la tabla 2, se muestra el número de beneficiarios directos e indirectos del proyecto. 
Tabla 2. Número de beneficiarios directos e

indirectos

\begin{tabular}{|c|c|c|}
\hline \multicolumn{3}{|l|}{ Número de Beneficiarios } \\
\hline Asociaciones / personas naturales bajo relación de dependencia/ comerciantes & Directos & Indirectos \\
\hline Otto Arosemena Gómez & 6 & 24 \\
\hline Anda Aguirre & 6 & 24 \\
\hline Unidad Educativa Guillermo Durán Arcentales & 21 & 84 \\
\hline Unidad Educativa "Río Amazonas" & 23 & 92 \\
\hline Unidad Educativa "Liceo Cristiano Milagro" & 11 & 44 \\
\hline Asociación de Agricultores Mariscal Sucre (Asgrimasu) & 25 & 100 \\
\hline Mercado Central de Milagro & 25 & 100 \\
\hline Asociación de productores agropecuarios La Lejía "ASPROLEJI" & 40 & 160 \\
\hline Asociación Agropecuaria General Pedro J. Montero & 16 & 64 \\
\hline Asociación Agropecuaria Maravillas Dos & 22 & 88 \\
\hline Sindicato General de Trabajadores de la Compañía Tropical Packing S.A & 50 & 200 \\
\hline Cooperativa de producción agrícola La Indiana & 16 & 64 \\
\hline Asociación de Trabajadores autónomos "La Paquita" & 24 & 96 \\
\hline Asociación de Emprendedores de producción y comercialización "Nueva Era" & 8 & 32 \\
\hline Junta Cantonal de Defensa del Artesano & 30 & 120 \\
\hline TOTAL & 323 & 1292 \\
\hline
\end{tabular}

Fuente: Proyecto de vinculación

Es importante precisar que esta encuesta está encaminada a conocer el nivel de cultura tributaria del beneficiario, su comportamiento tributario y el grado de aceptación de capacitarse en áreas tributarias y contables. Luego de aplicar la encuesta se obtuvieron los siguientes resultados.

En la formación tributaria por parte de los estudiantes de la carrera de Contaduría Pública y Auditoria de la UNEMI, participaron 143 hombres y 180 mujeres, mostrando que el grupo de beneficiarios en su mayoría son mujeres con un 56\% (Tabla 3)

Tabla 3. Género de los beneficiarios

\begin{tabular}{ccc}
\hline ALTERNATIVAS & RESULTADOS & PORCENTAJE \\
\hline Hombres & 143 & $44 \%$ \\
Mujeres & 180 & $56 \%$ \\
\hline TOTAL & $\mathbf{3 2 3}$ & $\mathbf{1 0 0 \%}$ \\
\hline
\end{tabular}

Fuente: Encuesta

De acuerdo a la tabla 4, la edad de los beneficiarios se concentra en rangos de 30 a 64 años que representa el $63 \%$ de los socios, $24 \%$ tienen edades entre 15 y 29 años, seguidos por el $13 \%$ que representa a aquellos participantes con rangos de edad mayores a 65 años.

Tabla 4. Edad de los beneficiarios

\begin{tabular}{ccc}
\hline ALTERNATIVAS & RESULTADOS & PORCENTAJE \\
\hline Menores de 15 años & 0 & $0 \%$ \\
De 15 a 29 años & 76 & $24 \%$ \\
De 30 a 64 años & 204 & $63 \%$ \\
De 65 y más años & 43 & $13 \%$ \\
\hline TOTAL & $\mathbf{3 2 3}$ & $\mathbf{1 0 0 \%}$ \\
\hline
\end{tabular}

Fuente: Encuesta

El $31 \%$ de los socios terminaron sus estudios secundarios y un $24 \%$ sus estudios primarios. En la tabla 5, se observa que el $27 \%$ de los beneficiarios tienen estudios de tercer nivel y $1 \%$ formación de postgrado esto se debe a que el proyecto capacitó a docentes de instituciones de educación media. Además, se observa que, un $12 \%$ y 5\% corresponde a la formación de artesanos $\mathrm{y}$ estudios en institutos tecnológicos 
respectivamente.

Tabla 5. Nivel de estudio de los beneficiarios

\begin{tabular}{ccc}
\hline ALTERNATIVA & RESULTADOS & PORCENTAJE \\
\hline Primaria & 78 & $24 \%$ \\
Secundaria & 100 & $31 \%$ \\
Artesano & 39 & $12 \%$ \\
Instituto tecnológico & 15 & $5 \%$ \\
Tercer nivel & 87 & $27 \%$ \\
Postgrado & 4 & $1 \%$ \\
\hline TOTAL & $\mathbf{3 2 3}$ & $\mathbf{1 0 0 \%}$ \\
\hline
\end{tabular}

Fuente: Encuesta

El $76 \%$ de los encuestados se dedican a actividades empresariales en las que prevalece la actividad de agricultura, $21 \%$ realiza actividades de relación de dependencia como la prestación de servicios de docencia, solo el $2 \%$ se dedica a exportar sus productos agrícolas (Tabla 6)

Tabla 6. Actividad económica de los beneficiarios

\begin{tabular}{|c|c|c|}
\hline ALTERNATIVAS & RESULTADOS & PORCENTAJES \\
\hline Actividad Empresarial & 247 & $76 \%$ \\
\hline Relación de Dependencia & 68 & $21 \%$ \\
\hline Importador & 0 & $0 \%$ \\
\hline Exportador & 8 & $2 \%$ \\
\hline TOTAL & 323 & $100 \%$ \\
\hline
\end{tabular}

Fuente: Encuesta

De la encuesta realizada se analiza el control tributario - contable de los beneficiarios, el $37 \%$ no tiene ningún control mostrando que no llevan registros de los ingresos y gastos del negocio y que, carecen de un apropiado control de las fechas y montos por los cuales deben cancelar impuestos. El 35\% de los socios asumen el control de su negocio y el 28\% contratan a profesionales para el manejo de sus movimientos económicos (Tabla 7).

Tabla 7. Control tributario - contable de los beneficiarios

\begin{tabular}{ccc}
\hline ALTERNATIVAS & RESULTADOS & PORCENTAJES \\
\hline Propietario & 112 & $35 \%$ \\
Profesional & 90 & $28 \%$ \\
Ningún control & 121 & $37 \%$ \\
\hline TOTAL & $\mathbf{3 2 3}$ & $\mathbf{1 0 0 \%}$ \\
\hline
\end{tabular}

Fuente: Encuesta

Con respecto a la preparación en temas tributarios - contables el 95\% de los encuestados considera que prepararse en estos temas contribuirá a su gestión administrativa e impulsará su negocio, sin embargo, el 5\% restante considera que no es necesario capacitaciones en temáticas tributarias y contables (Tabla 8)

Tábla 8. Preparación en temas tributarios - contables de los beneficiarios

\begin{tabular}{ccc} 
ALTERNATIVAS & RESULTADOS & PORCENTAJES \\
\hline $\mathrm{Si}$ & 307 & $95 \%$ \\
$\mathrm{No}$ & 16 & $5 \%$ \\
\hline TOTAL & $\mathbf{3 2 3}$ & $\mathbf{1 0 0 \%}$ \\
\hline
\end{tabular}

Fuente: Encuesta 
El registro de los contribuyentes en las bases de la Administración Tributaria es importante, los encuestados en un 53\% se encuentra inscrito en el RUC, 22\% tiene calificación RISE, y el 25\% de los socios afirma no tener registro en el SRI (Tabla 9)

Tabla 9. Control tributario - contable de los beneficiarios

\begin{tabular}{ccc}
\hline ALTERNATIVAS & RESULTADOS & PORCENTAJES \\
\hline RUC & 171 & $53 \%$ \\
RISE & 71 & $22 \%$ \\
No tiene registro & 81 & $25 \%$ \\
\hline TOTAL & $\mathbf{3 2 3}$ & $\mathbf{1 0 0 \%}$ \\
\hline
\end{tabular}

Fuente: Encuesta

En la segunda fase de vinculación, para evidenciar el efecto positivo de la capacitación se tomó una prueba de conocimientos a los beneficiarios de cuatro asociaciones: Mercado Central de Milagro, Asociación de Agricultores Mariscal Sucre (Asgrimasu), Asociación de productores agropecuarios La Lejía "ASPROLEJI" y la Asociación Agropecuaria General Pedro J. Montero.

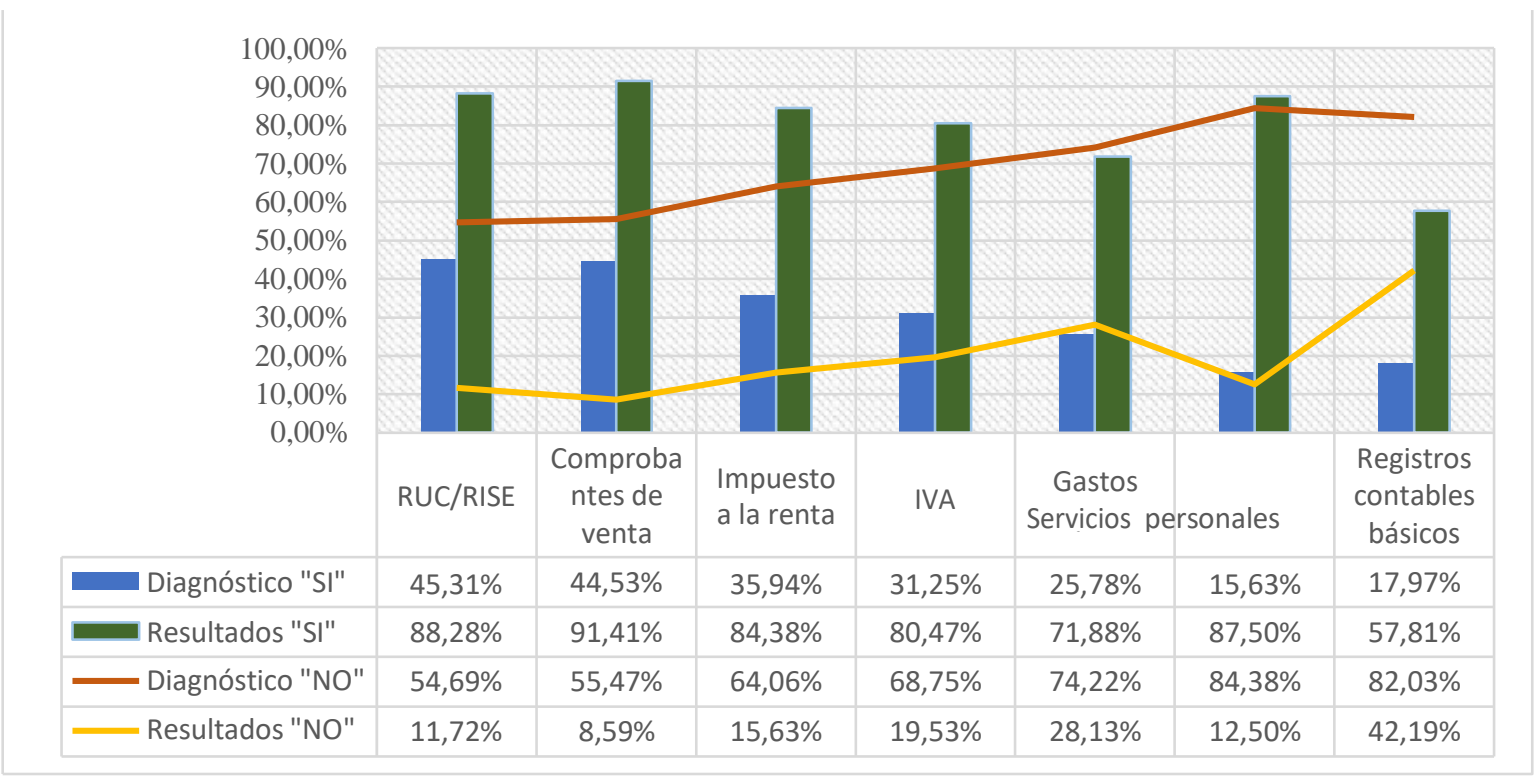

Figura 3. Capacitaciones en temas tributarios y ontables Fuente: encuestas y pruebas

Mediante las pruebas aplicadas a los beneficiarios objeto de análisis para determinar el cumplimiento del objetivo del proyecto se observa en la figura 3 que, por un lado, en la fase de diagnóstico los conocimientos en aspectos básicos del RUC/RISE son representativos en las asociaciones objeto de análisis con un 45,31\%. En segundo lugar, los beneficiarios conocen sobre comprobantes de ventas con un 44,53\% y le siguen el conocimiento del manejo del impuesto a la renta y del IVA con un 35,94\% y 31,25 \% respectivamente. Así también, se observa que en la fase de diagnóstico el tema que menos se domina son los servicios en línea con un $84,38 \%$ y los registros contables básicos con un $82,03 \%$.

Al aplicar las pruebas y los talleres luego de efectuar la capacitación se observa un incremento entre la fase de diagnóstico y la fase de resultados, aunque no refleja el $100 \%$ si se evidencia un mayor dominio de temas tributarios. En lo que respecta a temas como gastos personales y servicios en línea reflejan un $71,88 \%$ y un $87,50 \%$ respectivamente demostrando un incremento en sus conocimientos, aunque no es así en el manejo de registros contables básicos con un $57,81 \%$. 


\section{DISCUSIÓN}

Este proyecto permitió ayudar a la comunidad que por falta de conocimientos tributarios no declaran a tiempo los impuestos, trayendo como consecuencia el que se determinen sanciones, como intereses y multas por mora tributaria, afectando directamente al contribuyente y al Estado por no realizar las recaudaciones tributarias a tiempo, disminuyendo directamente el Presupuesto General, el mismo que se torna insuficiente no solo para que el gobierno de turno cumpla con las obras necesarias para el desarrollo del país, sino también a los contribuyentes en general, quienes serán observados con sanciones determinantes que establece la ley, como clausuras de establecimientos y multas pecuniarias, emitidas por los organismos de control.

El proyecto presenta un $95 \%$ de aceptación por parte de los miembros de las asociaciones para ejecutar formaciones tributarias. Con esto se pretende fomentar el cumplimiento de las obligaciones con el fisco, con la finalidad de crear conciencia tributaria en los beneficiarios y contribuir con la meta del PND 2017-2021, que es incrementar la recaudación de tributos.

Para la carrera de Contaduría Pública y Auditoría es una ventaja profesional porque permite a los estudiantes producir informes y documentos necesarios en las diversas actividades tributables para proporcionar un historial del cumplimiento de la legislación tributaria ecuatoriana y su correcta aplicación en la toma de decisiones. Además, le permite integrarse proactivamente al trabajo en equipo, asesorar con criterio técnico para la toma de decisiones oportuna, observa respeto a las diferencias culturales, a la biodiversidad y apoya con su ejercicio profesional a la comunidad en general.

Los beneficiarios oscilan entre los 30 y 64 años de edad, donde se observa poca presencia de jóvenes y, por tanto, mayor resistencia de los sectores vulnerables al cancelar sus impuestos, existiendo pagos a destiempo que surge por el desconocimiento de las obligaciones fiscales, siendo evidente en las poblaciones del área rural, donde están ubicados los miembros de las asociaciones.

La formación dirigida a las personas naturales no obligadas a llevar contabilidad en temas tributarios debe replicarse, de manera gratuita, para fortalecer el cumplimiento de los deberes formales, a través de convocatorias masivas de contribuyentes que deseen formarse para que los estudiantes transmitan los conocimientos adquiridos en las aulas de clases.

Los proyectos de vinculación deberían planificarse entre los gobiernos seccionales y las instituciones de educación superior con la finalidad de identificar las necesidades de los sectores vulnerables, llegar a más miembros de la comunidad y disponer de la infraestructura adecuada para satisfacer las necesidades de los beneficiarios. Por ello, el proyecto no solo debe ejecutarse por las instituciones educativas sino también por organismos de control y municipios, siendo importante crear alianzas estratégicas para contribuir con la recaudación del estado.

Las universidades y gobiernos seccionales deberían tener contacto constante con los beneficiarios, de tal forma que se pueda evaluar el impacto de los proyectos de vinculación y generar nuevas temáticas para proporcionar las herramientas necesarias a los sectores vulnerables y contribuir a su desarrollo. 


\section{REFERENCIAS BIBLIOGRÁFICAS}

Amasifuen, M. (2015). Importancia de la cultura tributaria en el Perú. Revista Accounting Power for Business, 1(1), 73-90. https://doi.org/2414-8180

Arriaga Baidal, G., Reyes Tomalá, M., Olives Maldonado, J., \& Solórzano Méndez, V. (2017). Análisis de la cultura tributaria: Impuesto a la renta para personas naturales no obligadas a llevar contabilidad, provincia de Santa Elena. Ciencias Pedagógicas E Innovación UPSE, (3), 118-127. https://doi.org/10.26423/rcpi.v5i3.192

Camarero, L., Pino, J., \& Mañas, B. (2015). Evolución de la cultura tributaria, coyuntura económica y expectativas vitales. Un estudio Longitudinal.

Cepal. (2018). Agenda 2030 y los Objetivos de Desarrollo Sostenible Una oportunidad para América Latina y el Caribe Gracias por su interés en esta publicación de la CEPAL. (Naciones Unidas, Ed.). Retrieved from https://repositorio.cepal.org/bitstream/handle/11362/40155/10/S1700334_es.pdf

CEPAL, OEI, \& Iberoamericana, S. G. (2010). 2021 Metas Educativas. La educación que queremos para la generación de los bicentenarios (OEI). Madrid.

INEC. (2018). Encuesta Nacional de Empleo, Desempleo y Subempleo (ENEMDU) Septiembre 2018. Quito.

Onofre-Zapata, R. F., Aguirre-Rodriguez, C. G., \& Murillo-Torres, K. G. (2017). La cultura tributaria y su incidencia en la recaudación de los tributos en el Cantón Babahoyo Ecuador. Revista Cientifica Dominio de Las Ciencias, 3(2477-8818), 3- 23. https://doi.org/10.23857/dom.cien.pocaip.2017.3.3.jun.45-68

Quintanilla, J. (2012). La universidad en la cultura tributaria. Retos, 2(3), 105-114. Retrieved from https://bit.ly/2i4ifHq

Secretaría Nacional de Planificación y Desarrollo. (2017). Plan Nacional de Desarrollo 2017-2021. https://doi.org/10.1109/CDC.2014.7039974

Servicio de Rentas Internas. (2018). Saiku - Next Generation Open Source Analytics. Retrieved January 26, 2019, from https://declaraciones.sri.gob.ec/saiku-ui/

UNESCO. (1998). Declaración mundia sobre la educación superior en el siglo XXI: visión y acción. Retrieved January 26, 2019, from http://www.unesco.org/education/educprog/wche/declaration_spa.htm

Van Der Bijl, B. (2015). La evaluación de carreras universitarias en el Ecuador ¿Desde qué concepción de educación? Estudio documental y bibliográfico Informe final, 922.

Retrieved from https://universidadsociedadec.files.wordpress.com/2015/03/informe-final-estudioceaaces.pdf 